\title{
Evaluation of Anti-Inflammatory Effect of Wedelolactone on Indomethacin Induced Colitis in Rats: Involvement of IL-6/STAT3 Pathway
}

\author{
Prakash Tigari 1,*(D), Suresh Janadri 1 ${ }^{10}$, Krishnadas Madhu ${ }^{1}$ (D), Nageena Taj ${ }^{1(\mathbb{D})}$ \\ 1 Department of Pharmacology, Acharya \& BM Reddy College of Pharmacy, Bengaluru, Karnataka, 560107 India; \\ prakashtigari@gmail.com (P.T.); sureshjanadri@acharya.ac.in (S.J.); krishnadas.madhu77@gmail.com (K.M.); \\ nageenataj@acharya.ac.in (N.T.); \\ * Correspondence: prakashtigari@gmail.com (P.T.);
}

Scopus Author ID 19639232300

Received: 28.04.2021; Revised: 6.06.2021; Accepted: 10.06.2021; Published: 8.08.2021

\begin{abstract}
The present study was carried out to study coumestan derivative wedelolactone in Indomethacin-induced enterocolitis in rats. Wistar rats were randomly divided into three groups containing six animals per group. Group I served as normal control. Group II, Group III \& Group IV receive $7.5 \mathrm{mg} / \mathrm{kg}$, s.c, indomethacin on two consecutive days. Group III and Group IV have received a wedelolactone dose of $50 \mathrm{mg} / \mathrm{kg}$, and $100 \mathrm{mg} / \mathrm{kg}$ per oral, respectively, for 14 days after the induction with indomethacin. The protective effect was measured based on intestinal parameters of the disease activity index, colitis score, myeloperoxidase (MPO) activity in the colon. The inflammation biomarkers were quantified by ELISA in the rat colon. Further, activity was ascertained by histopathology. Pro-inflammatory functions IL-1a, IL-1b, IL-2, TNF, INF $\gamma$, STAT3, and CCL-5 play an important role in the variation of the intestinal immune system. Wedelolactone showed significantly decreased Disease activity index, Colitis score, Myeoloperoxidase activity. Expression of proinflammatory was increased in indomethacin-induced groups and was significantly suppressed in animals administered with wedelolactone at $50 \mathrm{mg} / \mathrm{kg} \& 100 \mathrm{mg} / \mathrm{kg}$ dose $(\mathrm{p}<0.01 \& \mathrm{p}<0.001)$. Histological reports also revealed that treated groups have comparatively less damage than that of the induced groups. We concluded that wedelolactone showed an anti-inflammatory effect by downregulation of the IL-6/STAT3 inflammatory signaling pathway and the equilibrium production of pro-inflammatory cytokines.
\end{abstract}

Keywords: Wedelolactone; Indomethacin; colitis; Pro-inflammatory cytokines; IL-6/STAT3 pathway.

(C) 2021 by the authors. This article is an open-access article distributed under the terms and conditions of the Creative Commons Attribution (CC BY) license (https://creativecommons.org/licenses/by/4.0/).

\section{Introduction}

Crohn's disease (CD) and ulcerative colitis (UC) are denoted as inflammatory bowel disease (IBD) and chronic inflammatory disorders of the gastrointestinal tract. The pathogenesis of IBD has profoundly enhanced in recent years based in part on the use of experimental models of IBD [1].

Administration of subcutaneous (SC) injections of indomethacin induce a severe enteropathy in rats similar to human Crohn's disease [1]. Administration of indomethacin increases mucosal permeability, produces injury and inflammation in the small intestine. These symptoms consider Crohn's disease research model and could be used for new drug 
investigation [2]. Enterohepatic circulation of indomethacin is considered key in promoting the acute phase of the disease, while endogenous microflora is thought to play a role in the perpetuation and intensification of the chronic phase [3].

The onset of disease arises rapidly upon administration of indomethacin. Abrasions occur as longitudinal ulcers typically on the mesenteric side of the distal small intestine with variable degrees of inflammation and necrosis evident in all layers of the intestinal wall. A single SC injection $(7.5 \mathrm{mg} / \mathrm{kg}$ ) was revealed to cause acute injury and inflammation [1].

Various preclinical IBD models include (but not limited to) inflammation score assessment, PK/PD blood collection, measurement of body weight, intestinal immunohistochemistry images, MPO (myeloperoxidase) activity assessment, proinflammatory cytokine estimation including IL-1 $\beta$, IL-6, IFN-ÿ, TNF- $\alpha$, endoscopy imaging, inflammation-related miRNA study.

Wedelolactone (Figure 1) is a coumestan on an organic chemical compound found in Eclipta alba and Wedelia calendulace belongs to the phytoestrogen group of flavonoids. In our earlier study, HPLC and LC-MS spectra were used to isolate and classify wedelolactone from the Wedelia calendulace plant [4]. Kobori and co-workers [5] reported that wedelolactone, a natural compound that hinders the expression of LPS-induced caspase-11 in cultured cells by inhibiting transcription pathway NF-kappa B. Yuan and co-workers [6] reported that wedelolactone immensely inhibited the expression of iNOS and Cox-2 in LPS-stimulated cells by pro-inflammatory mediators, as well as downstream products like NO, PGE2 and TNF $\alpha$ [6]. Wedelolactone is well-known for inhibiting antioxidant, anti-inflammatory, and 5lipoxygenase $[7,8]$. Wedelolactone, a botanical compound, has anti-inflammatory effects that strongly down-regulate the expression of c-Myc mRNA in prostate cancer cells [9]. However, it remains unclear if wedelolactone will effectively ameliorate bowel inflammation. The object of the present study was to explore the effects of wedelolactone on inflammation of the intestines.

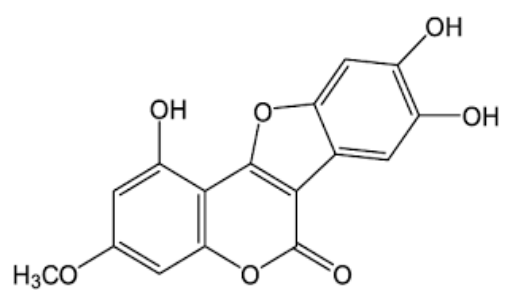

Figure 1. Chemical structure of Wedelolactone.

\section{Material and Methods}

\subsection{Animals.}

Female Albino Wistar rats from Biogen Laboratory Animal Facility, Bangalore, weighing 150-180 g, were procured. Animals were placed in a polypropylene cage and kept under a 12-h light / dark cycle at room temperature of $24 \pm 2^{\circ} \mathrm{C}$. Approval was received from the Committee on Institutional Animal Ethics (Ref: IAEC/ABMRCP/2016-17/PR/09), Acharya BM Reddy Pharmacy College, Bangalore. The animals had been fed a normal diet prior to and during the research, and their weights were recorded regularly. The animals were received water ad libitum. The animal studies were carried out in compliance with the guidelines of the CPCSEA and IAEC. 


\subsection{Drug, chemicals and ELISA kits.}

Wedelolactone (Baoji Guokang Bio-Technology CO., LTD, China), ELISA Kit IL-1a, IL-1b, IL-2, IL-6 INF-G, TNF-a, NFKB, CCL-5 (Lab Pro, Bangalore \& Krishgen BioSystems, Mumbai). All other reagents and chemicals were procured from local vendors and are analytical grade.

\subsection{Dose of Wedelolactone.}

Our previous reports selected Wedelolactone 50 and $100 \mathrm{mg} / \mathrm{kg}$ body weight for the present study $[10,11]$.

\subsection{Experimental design.}

The rats were randomly divided into three groups and consist of six rats. Group-I was a normal group and saline $(1 \mathrm{~mL} / \mathrm{kg}$, po) was administered continuously for 14 days through orogastric gavages. Group-II served as a chronic colitis control group and Group-III and Group IV served as treatment group received freshly prepared $7.5 \mathrm{mg} / \mathrm{kg}$ of indomethacin drug dissolved in 5\% sodium bicarbonate and administered at a dose of $0.5 \mathrm{ml}$ volume subcutaneously for consecutive 2 days. Wedelolactone (50 and $100 \mathrm{mg} / \mathrm{kg}$, po) was administered to Group-III and IV from the $3^{\text {rd }}$ day to the $14^{\text {th }}$ day through orogastric gavages [12]. Regular feed and water ad libitum were mentioned to all the groups up to the $14^{\text {th }}$ day.

\subsection{Disease activity index (DAI) and colitis score.}

According to a previously reported method [13], the rats were scored on day 1, day 3 and day 14 with respect to body weight, stool formation, and bloody stolen. To assess the disease's operation index, weight loss, the severity of stool formation, and bloody stool scores were averaged. The disease activity index and colitis scoring criteria are represented in Tables 1 and $2[14,15]$. The total colitis score of experimental groups was recorded on day 15.

Table 1. Disease activity index score (DAI).

\begin{tabular}{c|c|c|c} 
Score & $\begin{array}{c}\text { Decrease in growth } \\
(\boldsymbol{\%})\end{array}$ & Stool consistency & Gross rectal bleeding \\
\hline 0 & 0 & Normal & Normal \\
\hline 1 & $1-25$ & -- & -- \\
\hline 2 & $26-50$ & Loose stools & Fectawin $(+)$ \\
\hline 3 & $51-75$ & -- & Gross bleeding
\end{tabular}

Table 2. Colitis score calculated according to following criteria.

\begin{tabular}{c|l} 
Score & \multicolumn{1}{c}{ Appearance } \\
\hline 0 & No damage \\
\hline 1 & Localized hyperemia, no ulcers \\
\hline 2 & Ulceration without hyperemia or bowel wall thickening \\
\hline 3 & Ulceration with inflammation at one site \\
\hline 4 & Two or more sites of ulceration/inflammation \\
\hline 5 & Major sites of damage extending more than $1 \mathrm{~cm}$ along the length of the colon \\
\hline $6-10$ & $\begin{array}{l}\text { If the damage extends more than } 2 \mathrm{~cm} \text { along the length of the colon, the score is } \\
\text { increased by one for each additional } 1 \mathrm{~cm}\end{array}$
\end{tabular}

\subsection{Tissue homogenization.}

On day 15, all animals were then sacrificed by decapitation, and then colon tissues were isolated using sterile forceps and sterile scissors. The entire colon of each rat was detached by 
a ventral midline incision and opened longitudinally, washed immediately in chilled physiological saline, blotted and weight was recorded. Colon tissue $(500 \mathrm{mg})$ was placed in microcentrifuge tubes in $0.1 \mathrm{M}$ Phosphate Buffer saline $(\mathrm{pH} 7.4)$ solution and stored at $-80^{\circ} \mathrm{C}$ for Myeloperoxidase assay and $500 \mathrm{mg}$ of the tissue was homogenized in chilled lysis buffer. The homogenates were centrifuged $8000 \mathrm{xg}$ at $4^{\circ} \mathrm{C}$, for $15 \mathrm{~min}$. The collected supernatant was stored at $-80{ }^{\circ} \mathrm{C}$.

\subsection{Myeloperoxidase activity determination.}

Infiltration of neutrophils to the swollen colon was quantities indirectly using an MPO assay and expressed in ng/ml. MPO activity was determined by the modified method. The colon tissues were homogenized in $0.5 \%$ hexadecyl trimethyl ammonium bromide 0.5 mL50 $\mathrm{mg}$ of colon tissue; then the homogenates were centrifuged at $18,000 \mathrm{~g}$ for $15 \mathrm{~min}$ at $4^{\circ} \mathrm{C}$. Aliquots of $40 \mathrm{~mL}$ supernatant were mixed with $60 \mu \mathrm{L}$ potassium phosphate buffer $(50 \mathrm{mmol}$, $\mathrm{pH}$ 6.0) with o-dianisidine dihydrochloride and hydrogen peroxide. MPO activity was found from the rate of absorbance alteration in $3 \mathrm{~min}$ at $460 \mathrm{~nm}$ and calculation was done using the following formula [16].

$$
\frac{U}{m l}=\frac{(A \Delta \times V t \times 4)}{(E \Delta t \times V S)}
$$

$A$ - Difference in absorbance, $V t$ - Total volume, E-Extinction coefficient, $\Delta t-$ Measuring time, Vs - Sample volume.

\subsection{Measurement of cytokines -enzyme-linked immunosorbent assay (ELISA).}

The tissue homogenate of the isolated colon was subjected to the estimation of cytokines. Pro-inflammatory cytokines (IL-1 $\alpha$, IL-1 $\beta$, IL-2, IL-6, TNF- $\alpha$, STAT3, IFN $\gamma$, $\mathrm{NF \kappa B}$ and CCL-5) present in the colon supernatants was estimated by Enzyme-linked immunosorbent assay (ELISA) and expressed as $\mathrm{ng} / \mathrm{ml}$. Estimations were done in accordance with the manufacturer's recommendations (Lab Pro, Bangalore \& Krishgen BioSystems, Mumbai). At the end, the ELISA plates were spectrophotometrically evaluated at $450 \mathrm{~nm}$ (TECAN, Switzerland). All samples were assayed in triplicate.

\subsection{Histopathological assessment of colitis severity.}

The colon tissues were fixed in $10 \%$ phosphate-buffered formalin, regularly processed for paraffin embedding, sliced at $5 \mu \mathrm{m}$ and stained with hematoxylin. The histopathological study considered parameters such as tissue loss/cell death, eosin for colonic architecture assessment, the severity of the injury and mucosal epithelial lesion, infiltration of lymphocytes, inflammation and mucosal injury. Longitudinal sections of the distal colon were assessed based on adapted criteria of Pellino [17] and Wang [18].

\subsection{Statistical Analysis.}

All the data are represented in mean values \pm SEM. Statistical analyses were performed using one-way ANOVA. p-value $<0.05$ was considered significant. Calculations were performed using GraphPad Prism (version 6.0; GraphPad Software Inc., San Diego, CA, USA). 


\section{Results and Discussion}

\subsection{Effect of Wedelolactone on bodyweight.}

Effects of wedelolactone on body weight in colitis rats as showed in Table 3 . The body weight in indomethacin-induced colitis rats was decreased about $6.72,15.73$, and $22.01 \%$ at 1 , 3 , and 14 days, respectively, when compared to that of the normal control treatment with wedelolactone increased the body weight in colitis rats $(\mathrm{p}<0.01)$.

Table 3. Effect of wedelolactone on bodyweight.

\begin{tabular}{l|c|c|c}
\multirow{2}{*}{ Treatment } & \multicolumn{3}{|c}{ Changes in body weight } \\
& \multicolumn{3}{|c}{ Mean \pm SEM } \\
\hline & Day 1 & Day 3 & Day 14 \\
\hline Normal Control & $173.33 \pm 6.14$ & $180.00 \pm 4.70$ & $181.67 \pm 4.04$ \\
\hline Indomethacin colitis control & $161.67 \pm 1.66$ & $151.67 \pm 3.07$ & $141.67 \pm 4.04$ \\
& $(6.72 \downarrow)$ & $(15.73 \downarrow)$ & $(22.01 \downarrow)$ \\
\hline Wedelolactone treatment group & $163.54 \pm 1.16$ & $151.97 \pm 4.56$ & $154.36 \pm 3.87$ \\
$(50 \mathrm{mg} / \mathrm{kg})$ & $(1.15 \uparrow)$ & $(0.19 \uparrow)$ & $(8.95 \uparrow)$ \\
\hline Wedelolactone treatment & $166.67 \pm 3.33$ & $153.33 \pm 2.11$ & $160.00^{* *} \pm 2.58$ \\
$(100 \mathrm{mg} / \mathrm{kg})$ & $(3.09 \uparrow)$ & $(1.09 \uparrow)$ & $(12.93 \uparrow)$
\end{tabular}

Group I-Normal group, Group II-Indomethacin induced colitis control, Group III-Wedelolactone treatment group (50 mg/kg) and Group IVWedelolactone treatment group $(100 \mathrm{mg} / \mathrm{kg}$. The values are expressed as Mean $\pm \operatorname{SEM}(\mathrm{n}=6)$. ** $<0.01, * * * \mathrm{p}<0.001$, Data was analyzed by One-Way Analysis of Variance (ANOVA) followed by Dunnett's test. In parenthesis, it showed \% of decreased $(\downarrow) /$ increased $(\uparrow)$ in body weight.

\subsection{Effect of Wedelolactone on DAI and colitis score.}

The DAI score is a common parameter used for evaluating colitis severity. Higher DAI score suggests a greater state of colitis. Compared with the normal control group, the DAI score of the indomethacin group was significantly increased, indicating that the indomethacin group showed substantial loss of body weight, diarrhea and bloody stool. Consistent with the findings from changes in body weight, the results from stool consistency score also found that colitis animals showed a raised score when compared to that of the control. Wedelolactone markedly decreased the stool consistency score. The DAI scores of the wedelolactone treated group were suppressed; however, the effect of wedelolactone at $100 \mathrm{mg} / \mathrm{kg}$ was considered statistically significant (Table 4). The colitis scoring was recorded on day 15 and data showed in Table 4, it was significantly increased in the indomethacin-induced colitis control group (colitis score was 9.6 out of 10 on day 15) compared to normal animals. The colitis score of wedelolactone $(100 \mathrm{mg} / \mathrm{kg})$ treated animals was 2.33 out of 10 on day 15 and significantly $(\mathrm{p}<0.001)$ decreased compared with indomethacin colitis control animals.

Table 4. Effect of wedelolactone on disease activity index (DAI) and colitis score of indomethacin-induced

\begin{tabular}{l|l|l|l|l}
\multicolumn{1}{c}{ Troups } & \multicolumn{1}{c}{ Treatment } & \multicolumn{1}{c}{ DAI (Day 3) } & DAI (Day 14) & \multicolumn{1}{c}{ Colitis Score } \\
\hline I & normal saline, & $00.00 \pm 00.00$ & $00.00 \pm 00.00$ & $00.00 \pm 00.00$ \\
\hline II & indomethacin $(7.5 \mathrm{mg} / \mathrm{kg})$ & $1.88 \pm 0.31$ & $2.77 \pm 0.26$ & $9.66 \pm 0.18$ \\
\hline III & $\begin{array}{l}\text { indomethacin }(7.5 \mathrm{mg} / \mathrm{kg}) \\
\text { wedelolactone }(50 \mathrm{mg} / \mathrm{kg})\end{array}$ & $2.218 \pm 0.41$ & $1.35 \pm 0.31 * *$ & $5.7 \pm 0.26 * * *$ \\
\hline IV & $\begin{array}{l}\text { indomethacin }(7.5 \mathrm{mg} / \mathrm{kg}) \\
\text { wedelolactone }(100 \mathrm{mg} / \mathrm{kg})\end{array}$ & $2.0 \pm 0.37$ & $0.99 \pm 0.28 * * *$ & $2.33 \pm 0.14 * * *$ \\
& & &
\end{tabular}

Group I-Normal group, Group II-Indomethacin induced colitis control, Group III-Wedelolactone treatment group (50 mg/kg) and Group IVWedelolactone treatment group $(100 \mathrm{mg} / \mathrm{kg}$. The values are expressed as Mean $\pm \operatorname{SEM}(\mathrm{n}=6) . * * \mathrm{p}<0.01, * * * \mathrm{p}<0.001$, Data was analyzed by One-Way Analysis of Variance (ANOVA) followed by Dunnett's test. 


\subsection{Effect of wedelolactone on myeloperoxidase.}

The colonic MPO activity denotes another index of inflammation. Myeloperoxidase enzymes were assessed in all experimental groups as a sign of neutrophil influx. Indomethacin administration resulted in increased MPO activity, indicative of neutrophil infiltration. The increase was significant $(\mathrm{p}<0.001)$ higher in colitis rats (Figure 2). However, wedelolactonetreated rats presented a decrease in MPO concentration at $100 \mathrm{mg} / \mathrm{kg}$ dose $(\mathrm{p}<0.01)$.

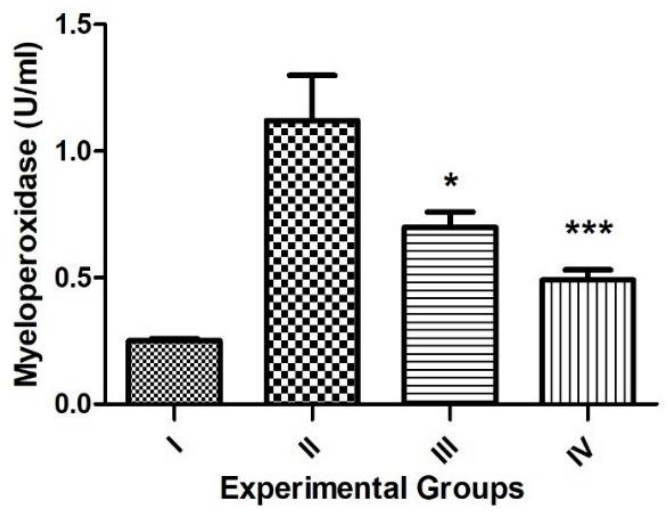

Figure 2. Effect of Wedelolactone on Myeloperoxidase assay (MPO) of indomethacin-induced. Group INormal group, Group II- indomethacin-induced colitis control, Group III-wedelolactone treatment group (50 $\mathrm{mg} / \mathrm{kg}$ ), Group IV-wedelolactone treatment group $(100 \mathrm{mg} / \mathrm{kg})$. The values are expressed as Mean $\pm \mathrm{SEM}$ $(\mathrm{n}=6) . * \mathrm{p}<0.05, * * \mathrm{p}<0.01$ and $* * * \mathrm{p}<0.001$, data was analyzed by one-way analysis of variance (ANOVA) followed by Dunnett's test.

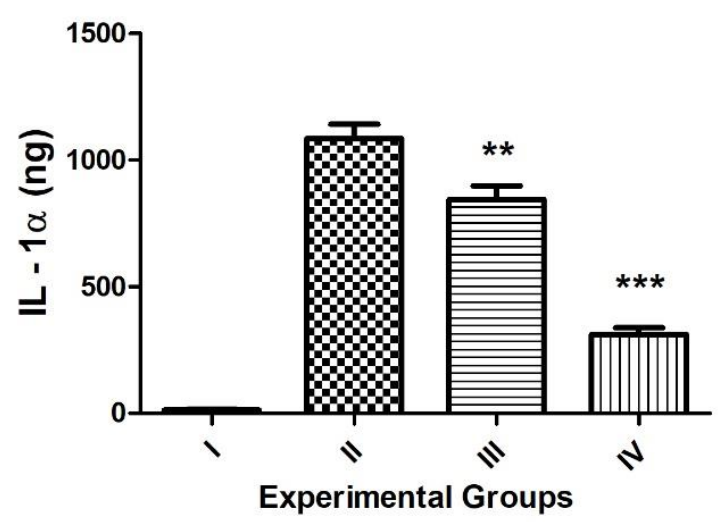

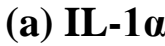

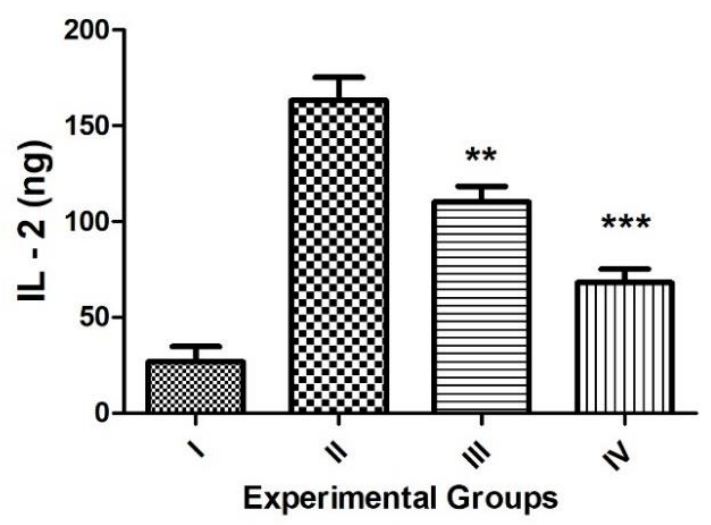

(c) IL-2

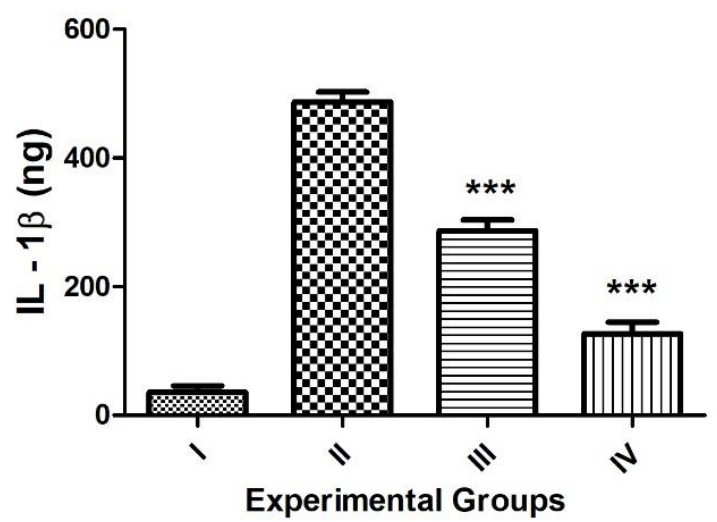

(b) IL-1及

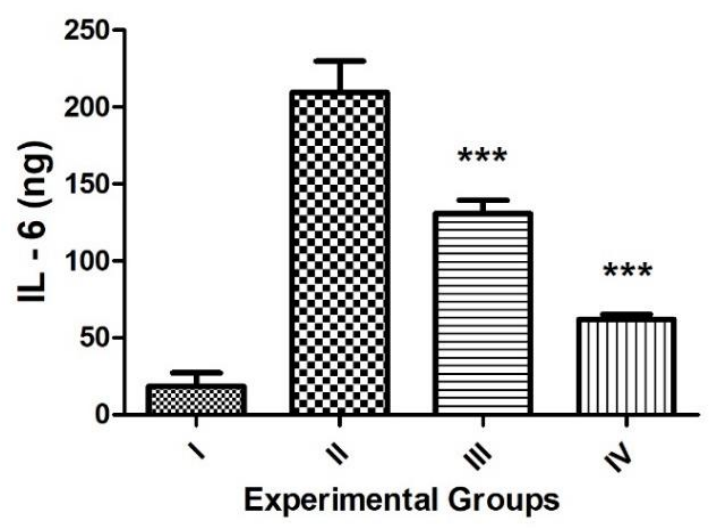

(d) IL-6 


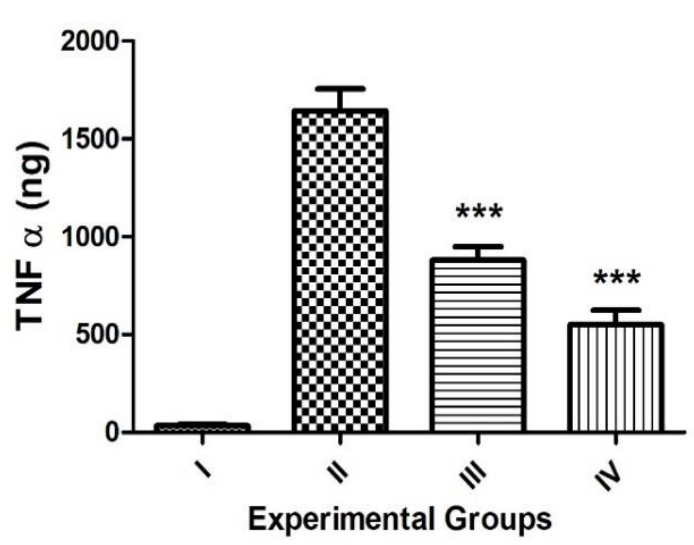

(e) TNF- $\alpha$

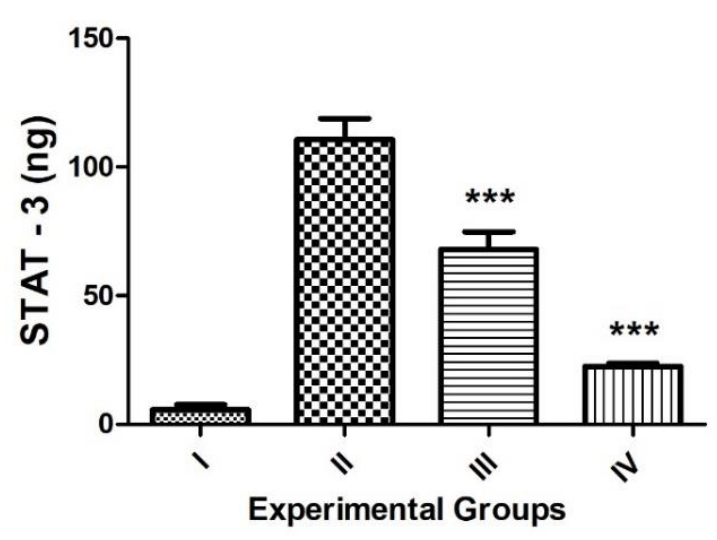

(g) STAT -3

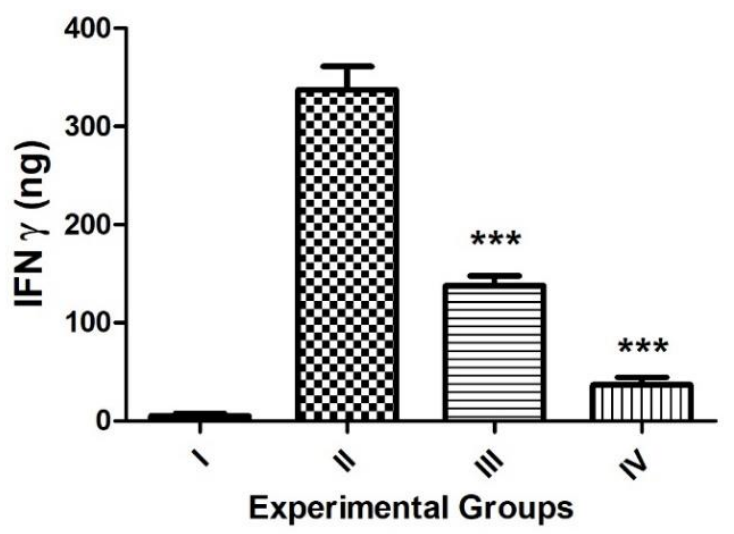

(f) IFN- $\gamma$

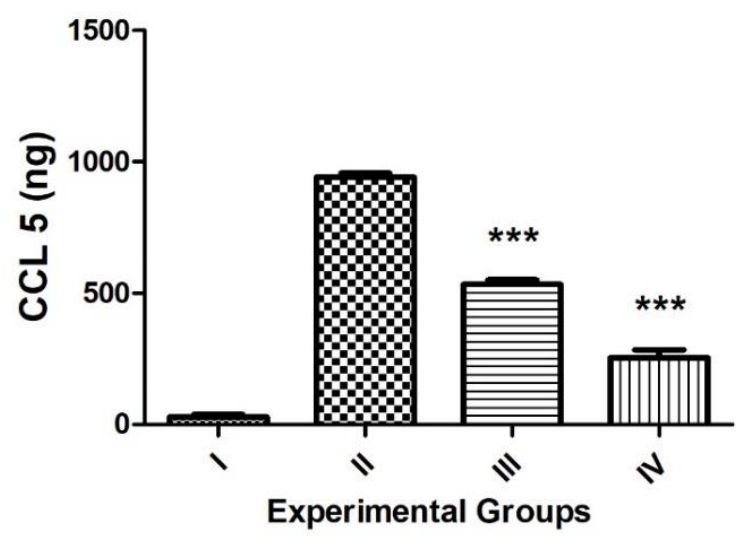

(h) CCL-5

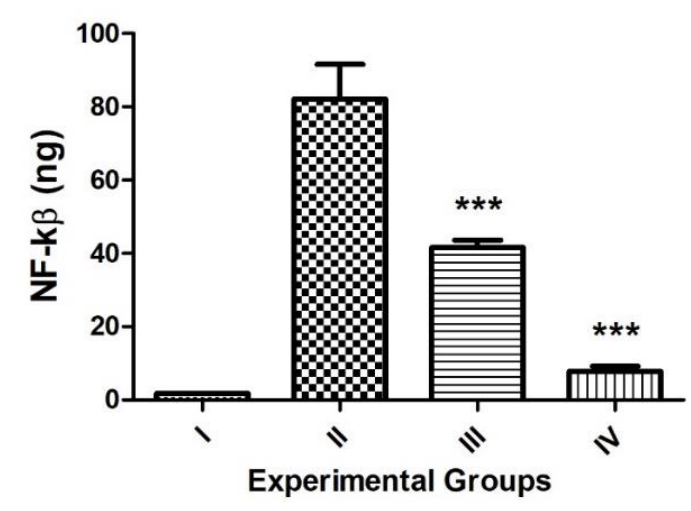

(i) $\mathrm{NF}-\mathrm{\kappa B}$

Figure 3. Effect of wedelolactone on pro-inflammatory cytokines $[(\mathbf{a}) \mathrm{IL}-1 \alpha$; (b) IL-1 $\beta$; (c) IL-2; (d) IL-6; (e) TNF- $\alpha$; (f) IFN $\gamma$; (g) STAT3; (h) CCL-5; (i) NFאB)] of indomethacin-induced colitis. Group I-Normal group, Group II- indomethacin-induced colitis control, Group III-wedelolactone treatment group (50 mg/kg), Group IV-wedelolactone treatment group $(100 \mathrm{mg} / \mathrm{kg})$. The values are expressed as Mean $\pm \mathrm{SEM}(\mathrm{n}=6)$. ${ }^{*} \mathrm{p}<0.05$,

$* * \mathrm{p}<0.01$ and $* * * \mathrm{p}<0.001$, Data was analyzed by one-way analysis of variance (ANOVA) followed by Dunnett's test.

\subsection{Effect of Wedelolactone on cytokine production in the colon.}

After experimentally colitis induction by indomethacin deregulated cytokines in pathological conditions promoting a pro-inflammatory effect, such as IL-1 $\alpha$, IL-1 $\beta$, IL-2, IL6 , TNF- $\alpha$, STAT3, IFN $\gamma$, NF $\kappa$ B and CCL-5. Rats with indomethacin-induced colitis revealed a significant increase in IL- $1 \alpha$, IL-1 $\beta$, IL-2, IL-6, TNF- $\alpha$, STAT3, IFN $\gamma$, NF $\kappa$ B and CCL-5 compared with the normal rats $(\mathrm{p}<0.001)$ indicates a systemic diseased condition (Figure 3a- 
4i). However, wedelolactone treated $(50 \& 100 \mathrm{mg} / \mathrm{kg})$ rats showed a significant decrease in pro-inflammatory cytokines compared with the indomethacin colitis group $(\mathrm{p}<0.01 \&$ $\mathrm{p}<0.001)$. These results showed that wedelolactone $100 \mathrm{mg} / \mathrm{kg}$ prevents inflammatory reaction by downregulating cytokine expression and data presented in Figure 3 (a - i).

\subsection{Histopathological assessment of colitis severity.}

Representative images for the analyzed study groups interpreting the histopathological score are shown in Figure 4. In short, the indomethacin exhibited significant hemorrhaging of diffuse transmural necrosis, involving mucosa, submucosa, muscle layer and serosa, and linked with peritonitis. Similar lesions were observed in the rats treated with wedelolactone, namely transmural necrosis but to a slighter degree and with a multifocal form, scattered with areas where the integrity of the mucosa was preserved. There was mild to moderate epithelial erosion and ulceration in these areas, and severe inflammatory cell infiltration was observed, extending to the submucosa. The normal group not exhibited epithelial erosion, inflammatory cell infiltration and lesions, epithelial erosion and ulceration.

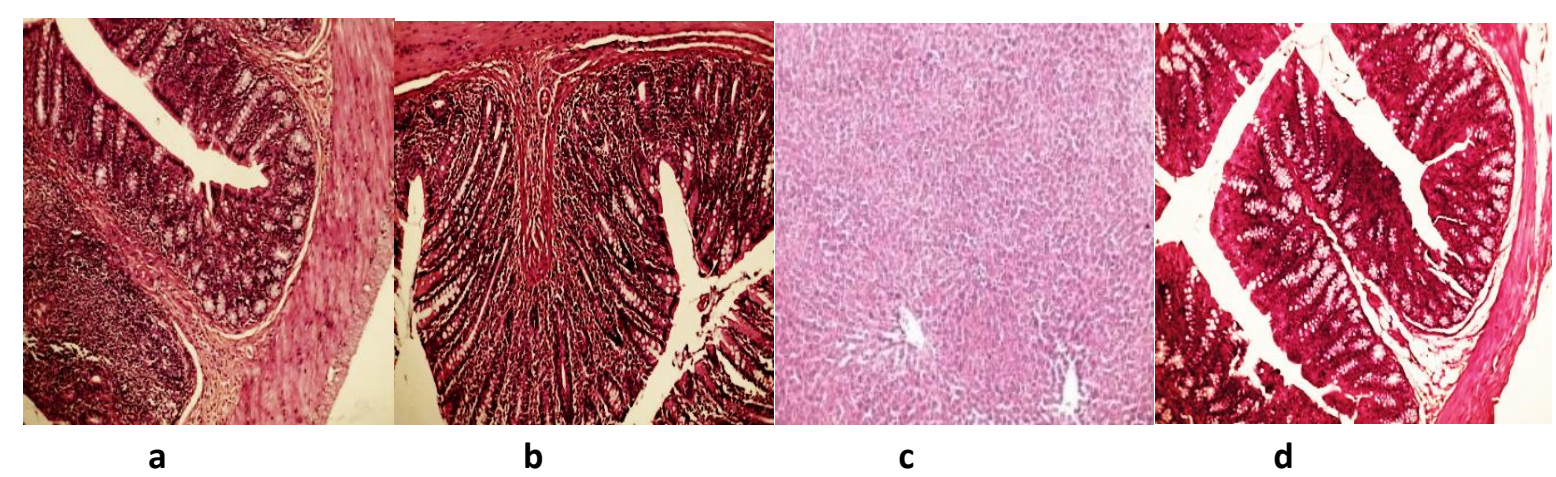

Figure 4. Histopathological changes in the experimental groups. Each image corresponding with a different experimental group, namely; (a) Group I-Normal group; (b) Group II-Indomethacin induced colitis control; (c)

Group III-Wedelolactone treatment group (50 mg/kg); (d) Group IV-Wedelolactone treatment group (100 $\mathrm{mg} / \mathrm{kg})$.

Animal models of intestinal inflammation are also used to study the pathogenesis of human IBD [19]. For example, models of colitis caused by indomethacin, TNBS, acetic acid, and dextran sulfate have been developed to explain the pathogenic and molecular mechanisms of human IBD 90 [20]. A model for animal colitis has generally been regarded as transmural granulomatous inflammation associated with diarrhea, rectal prolapse, weight loss and colonic wall thickening [21].

The role of cytokines in mucosal immunity continues to evolve as new information, cytokines, are considered signals of the mucosa-associated immune system. Gut immunological homeostasis is maintained by the interaction between host immunity and intestinal microbiota to keep gut homeostasis [22]. For normal gut homeostasis, the equilibrium of pro- and anti-inflammatory cytokines in the colonic mucosa is integral. The disruption of the cytokines in favor of pro-inflammatory cytokine increased production leads to disease and is observed in IBD [23]. The role of pro-inflammatory cytokines such as IL-1 $\alpha$, IL-1 $\beta$, IL-2, IL-6, TNF- $\alpha$, IFN $\gamma$, NFאB, STAT3 and CCL-5 in IBD is linked with the initiation and progression of ulcerative colitis and Crohn's disease.

Once a duodenal ulcer is caused by indomethacin or other NSAIDs, it has been suggested that the production of pro- and anti-inflammatory cytokines be a critical aspect of 
duodenal ulcer pathogenesis and etiology. In comparison, inflammatory responses play a key role in ulcerative colitis pathogenesis. Increased pro-inflammatory cytokines such as TNF- $\alpha$, IFN- $\alpha$, STAT3, CCL-5 and IL-1 $\alpha$, IL-1 $\beta$, IL-2, IL-6 intensify the inflammatory cascade and cause damage of the intestinal tissue in indomethacin-induced ulcerative colitis [24]. The increased expression of TNF- $\alpha$ in intestinal mucosal dysfunction is important among certain cytokines [25]. TNF- $\alpha$ is an essential pro-inflammatory cytokine of acute inflammation and is linked to the apoptosis of duodenal mucosal cells, which are destroyed by various agents [26]. Indomethacin administration stimulated the innate immune response and encouraged increased levels of TNF- $\alpha$ in the duodenal tissue, as shown by the concentration of TNF- $\alpha$ in the colitis control group. However, treated with wedelolactone, a significant decrease in TNF- $\alpha$ content was observed.

Additionally, IL-1 $\beta$ is a crucial mediator of ulcerative colitis progression. Inhibition of the action of IL-1 $\beta$ can decrease the severity of diarrhea and decrease inflammatory cell infiltration into the intestinal tissue [27].

In the present study, the levels of IFN- $\gamma$, TNF- $\alpha$, STAT3, CCL-5 and IL-1 $\alpha$, IL-1 $\beta$, IL-2, IL-6 were remarkably reduced by wedelolactone in indomethacin-induced colitis rats, indicating that the protecting effect of wedelolactone against colonic injury is linked to the downregulation of TNF- $\alpha$, IFN- $\gamma$, STAT3, CCL-5 and IL-1 $\alpha$, IL-1 $\beta$, IL-2, IL-6.

Chemokine (C-C motif) ligand 5 (CCL-5) is a CCL-5 gene-encoded protein in humans [28]. It is also known as RANTES (regulated on activation, expressed and secreted in normal T cells). CCL-5 is chemotactic for T cells, eosinophils, and basophils and shows an important role in leukocyte recruitment into inflammatory sites. CCL-5 plays an important role in the recruitment into inflammatory sites of many leukocytes, including $\mathrm{T}$ cells, macrophages, eosinophils, and basophils. In co-operation with certain cytokines produced by $\mathrm{T}$ cells such as IL-2 and IFN- $\gamma$ [29]. CCL-5 levels have risen in the colitis tissue, as demonstrated by the inflammation in the control group. However, a significant decrease in the CCL-5 parameter was observed in the wedelolactone-treated group.

The cause of ulcerative colitis, including genetics, environment and diet, is not fully understood as an idiopathic, colonic inflammatory disease of the mucosal. Of these risks, inflammation seemed a dynamic force of molecular changes in ulcerative colitis, i.e., IL6/STAT3 inflammatory signaling pathway has been reported to be involved in the mechanism and development of a number of inflammatory disorders. IL-6 signaling via STAT3 was a crucial LPS-driven pro-inflammatory response regulator, including TNF- $\alpha$, IL-1 $\alpha$, IL-6, IL-2, and STAT3 [30-33]. These pro-inflammatory mediators activated increased the inflammatory response and damaged the intestinal tissue. Activating the IL-6/STAT3 inflammatory signaling pathway also promoted an increase in endothelial monolayer permeability and brought a sustained loss of endothelial barrier function [34-38].

IL-6 is an immunoregulatory cytokine that activates IL-6 [39,40], consisting of a cell surface signaling assembly. In ulcerative colitis pathogenesis, IL-6 signaling via signal transducer and transcription activator-3 (STAT3) plays a main role, additionally in carcinogenesis of ulcerative colitis-associated colorectal cancers [41].

The lower neutrophil infiltration was confirmed biochemically, a decrease in colonic myeloperoxidase activity by wedelolactone in colonic tissue is also marked in histopathological observations, which confirms the decreased concentration of MPO in the inflamed colon after treatment. These results indicate that the beneficial effect of treatment with wedelolactone may be due in part to the suppression of the inflammatory response by leucocyte infiltration 
inhibition [42-44]. Ulcerative colitis is a highly infectious inflammatory condition characterized by weight loss, stomach pain, and bloody diarrhea. Wedelolactone treatment was able to attenuate weight loss, bloody diarrhea in the colitis rats. Moreover, wedelolactone also significantly reduced the disease index and colitis scores of rats during the experimental period.

\section{Conclusion}

Our research examined the possible role wedelolactone can play in ulcerative colitis anti-inflammation. Destruction of protective mucinous membrane resulted in epithelial cell damage and IL-6/STAT3 inflammatory signaling pathway expression upregulation in colorectal epithelial cells and ulcerative colitis in the end. The wedelolactone mechanism may involve the down-regulating the inflammatory signaling pathway IL-6/STAT3. The IL6/STAT3 pathway thus appeared to be the main node in the treatment of ulcerative colitis. Our results suggested that wedelolactone might block the IL-6/STAT3 inflammatory signaling pathway and exhibit an anti-inflammatory effect in ulcerative colitis.

\section{Funding}

This work was funded by the Rajiv Gandhi University of Health Science, Karnataka, Bangalore, India (Grant Order NO: RGU: RGU/ADV.RES/GRANTS/059/2016-17; www.rguhs.ac.in/AdvanceResearch.htm).

\section{Acknowledgment}

The authors are grateful to the Advanced Research Dept, Rajiv Gandhi University of Health Science, Karnataka, Bangalore, for financial support. We are thankful to the Principal and Management, Acharya Institution, Bangalore, for provided facilities.

\section{Conflicts of Interest}

The authors declare no conflicts of interest.

\section{Reference}

1. Mizoguchi, E.; Low, D.; Ezaki, Y.; Okada, T. Recent updates on the basic mechanisms and pathogenesis of inflammatory bowel diseases in experimental animal models. Intest Res. 2020 18, 151-167, https://doi.org/10.5217/ir.2019.09154.

2. Shanmugam, S.; Thangaraj, P.; Dos Santos Lima, B.; Trindade, G.G.G.; Narain, N.; Mara de Oliveira E Silva, A.; Santin, J.R.; Broering, M.F.; Serafini, M.R.; Quintans-Júnior, L.J.; Antunes de Souza Araújo, A. Protective effects of flavonoid composition rich P. subpeltata Ortega. on indomethacin induced experimental ulcerative colitis in rat models of inflammatory bowel diseases. J Ethnopharmacol 2020, 248, 112350, https://doi.org/10.1016/j.jep.2019.112350.

3. Ghafarzadeh, S,; Hobbenaghi, R,; Tamaddonfard, E; Farshid, A.A; Imani, M. Crocin exerts improving effects on indomethacin-induced small intestinal ulcer by antioxidant, anti-inflammatory and anti-apoptotic mechanisms. Vet Res Forum. 2019, 10, 277-284, https://doi.org/10.30466/vrf.2018.93512.2256.

4. Prakash, T.; Rama Rao, N.; Viswanath Swamy, A.H. Neuropharmacological studies on Wedelia

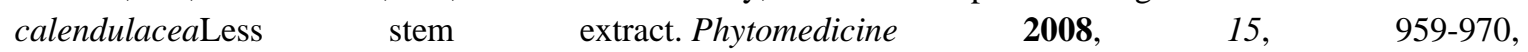
https://doi.org/10.1016/j.phymed.2008.05.005.

5. Kobori, M.; Yang, Z.; Gong, D.; Heissmeyer, V.; Zhu, H.; Jung, Y.K.; Gakidis, M.A.; Rao, A.; Sekine, T.; Ikegami, F.; Yuan, C.; Yuan, J. Wedelolactone suppresses LPS-induced caspase-11 expression by directly inhibiting the IKK complex. Cell Death Differ 2004, 11, 123-130, https://doi.org/10.1038/sj.cdd.4401325. 
6. Yuan, F.; Chen, J.; Sun, P.P.; Guan, S.; Xu, J. Wedelolactone inhibits LPS-induced pro-inflammation via NFkappaB pathway in RAW 264.7 cells. J Biomed Sci 2013, 20, 84-94, https://doi.org/10.1186/1423-0127-2084.

7. Xican, Li.; Tingting, Wang, Jingjing, Liu.; Yulong Liu.; Jun Zhang.; Jian, Lin. Zhongxiang Zhao, Dongfeng Chen. Effect and mechanism of wedelolactone as antioxidant-coumestan on OH-treated mesenchymal stem cells. Arab J Chem 2020, 13, 184-192, https://doi.org/10.1016/j.arabjc.2017.03.008.

8. Wagner, H.; Fessler, B. In vitro 5-lipoxygenase inhibition by Eclipta alba extracts and the coumestan derivative wedelolactone. Planta Med 1986, 52, 374-377, https://doi.org/10.1055/s-2007-969189.

9. Sarveswaran, S.; Ghosh, R.; Parikh, R.; Ghosh, J. Wedelolactone, an anti-inflammatory botanical, interrupts c-Myc oncogenic signaling and synergizes with enzalutamide to induce apoptosis in prostate cancer cells. Mol Cancer Ther 2016, 15, 2791-2801, https://doi.org/10.1158/1535-7163.MCT-15-0861.

10. Maya, S.; Prakash, T.; Divakar, Goli. Evaluation of neuroprotective effects of Wedelolactone and Gallic acid on aluminium-induced neurodegenaration: Relevance to sporadic Amyotrophic lateral sclerosis. Eur. J. Pharmacol 2018, 835, 41-51, https://doi.org/10.1016/j.ejphar.2018.07.058.

11. Maya, S.; Prakash, T.; Divakar, Goli.; Effect of Wedelolactone and Gallic acid on quinolinic acid induced neurotoxicity and impaired motor function: significance to sporadic amyotrophic lateral sclerosis. Neuro Toxicology 2018, 68, 1-12, https://doi.org/10.1016/j.neuro.2018.06.015.

12. Kawada, M.; Arihiro, A.; Mizoguchi, E. Insights from advances in research of chemically induced experimental models of human inflammatory bowel disease. World J Gastroenterol 2007, 13, 5581-5593, https://doi.org/10.3748/wjg.v13.i42.5581.

13. Xiao, H.T.; Lin, C.Y.; Ho, D.H.; Peng, J.; Chen, Y.; Tsang, S.W.; Wong, M.; Zhang, X.J.; Zhang, M.; Bian, Z.X. Inhibitory effect of the gallotannincorilagin on dextran sulfate sodium-induced murine ulcerative colitis. J Nat Prod 2013, 76, 2120-2125, https://doi.org/10.1021/np4006772.

14. Cooper, H.S.; Murthy, S.N.S.; Shah, R.S; Sedergran, D.J. P-659 - Clinicopathologic study of dextran sulfate sodium experimental murine colitis. Lab Invest 1993, 69, 238-249, https://doi.org/10.1016/S00215198(19)41298-5.

15. Damiani, C.R.; Benetton, C.A.; Stoffel, C.; Bardini, K.C.; Cardoso, V.H.; Di, Giunta G.; Pinho, R.A.; DalPizzol, F.; Streck ,E.L. Oxidative stress and metabolism in animal model of colitis induced by dextran sulfate sodium. J Gastroenterol Hepatol 2007, 22, 1846-1851, https://doi.org/10.1111/j.1440-1746.2007.04890.x.

16. Zhu, S.; Zeng, M.; Feng, G.; Wu, H. Platinum nanoparticles as a therapeutic agent against dextran sodium sulfate-induced colitis in mice. Int J Nanomedicine 2019, 14, 361-8378, https://doi.org/10.2147/IJN.S210655.

17. Pellino, G.; Keller, D.S.; Sampietro, G.M. Inflammatory bowel disease position statement of the Italian Society of Colorectal Surgery (SICCR): Crohn's disease. Tech Coloproctol 2020, 24, 421-448. https://doi.org/10.1007/s10151-020-02183-z.

18. Wang, H.; Vilches-Moure, J.G.; Cherkaoui, S.; Tardy, I.; Alleaume, C; Bettinger, T.; Lutz, A., Paulmurugan, R. Chronic Model of Inflammatory Bowel Disease in IL-10-/- Transgenic Mice: Evaluation with Ultrasound Molecular Imaging. Theranostics 2019, 9, 6031-6046, https://doi.org/10.7150/thno.37397.

19. Misselwitz, B.; Juillerat, P.; Sulz, M.C.; Siegmund, B.; Brand, S. Emerging Treatment Options in Inflammatory Bowel Disease: Janus Kinases, Stem Cells, and More. Digestion 2020, 101, 69-82, https://doi.org/10.1159/000507782.

20. Randhawa, P.K,; Singh, K,; Singh, N,; Jaggi, A.S. A review on chemical-induced inflammatory bowel disease models in rodents. Korean J Physiol Pharmacol 2014, 18, 279-288, https://doi:10.4196/kjpp.2014.18.4.279.

21. Lu, K.; Zhou, J.; Deng, J.; Li, Y.; Wu, C.; Bao, J. Periplaneta americana Oligosaccharides Exert AntiInflammatory activity through immunoregulation and modulation of gut microbiota in acute colitis mice model. Molecules. 2021, 26, 1718, https://doi.org/10.3390/molecules26061718.

22. Izadi, F,; Soheilifar, M.H,; Keshmiri Neghab H,; Soheilifar, M,; Esmaeeli Djavid, G. Network Analysis of Common Genes and Transcriptional Factors between Celiac Disease and Inflammatory Bowel Diseases. Middle East J Dig Dis. 2020, 12, 257-264, https://doi.org/10.34172/mejdd.2020.191.

23. Ramos, G.P.; Papadakis, K.A. Mechanisms of Disease: Inflammatory Bowel Diseases. Mayo Clin Proc 2019, 94,155-165, https://doi.org/10.1016/j.mayocp.2018.09.013.

24. Neurath, M.F. IL-23 in inflammatory bowel diseases and colon cancer. Cytokine Growth Factor Rev 2019, 45, 1-8, https://doi.org/10.1016/j.cytogfr.2018.12.002.

25. Biasi, F.; Leonarduzzi, G.; Oteiza, P.I.; Poli, G. Inflammatory bowel disease: mechanisms, redox considerations, and therapeutic targets. Antioxid Redox Signal 2013, 19, 1711-1747, https://doi.org/10.1089/ars.2012.4530. 
26. Tamaddonfard, E; Erfanparast, A.; Farshid, A.A.; Imani, M.; Mirzakhani, N.; Salighedar, R.; Tamaddonfard, S. Safranal. A constituent of saffron, exerts gastro-protective effects against Indomethacin-induced gastric ulcer. Life Sciences 2019, 224, 88-94, https://doi.org/10.1016/j.lfs.2019.03.054.

27. Ki, H.K.; Murakami, A.; Hayashi, R.; Ohigashi, H. Interleukin- $\beta$ targets interleukin-6 in progressing dextran sulfate sodium-induced experimental colitis. Biochem Biophys Res Commun 2005, 337, 647- 654, https://doi.org/10.1016/j.bbrc.2005.09.107.

28. Donlon, T.A.; Krensky, A.M.; Wallace, M.R.; Collins, F.S.; Lovett, M.; Clayberger, C. Localization of a human T-cell-specific gene, RANTES (D17S136E), to chromosome 17q11.2-q12" Genomics 1990, 6, 548553, https://doi.org/10.1016/0888-7543(90)90485-d.

29. Soria, G.; Ben-Baruch, A. The inflammatory chemokines CCL2 and CCL5 in breast cancer. Cancer Letters 2008, 267, 271-285, https://doi.org/10.1016/j.canlet.2008.03.018.

30. Adar, T.; Shteingart, S.; Ben-Ya'acov, A.; Shitrit, A.B.; Livovsky, D.M.; Shmorak, S.; Mahamid, M.; Melamud, B.; Vernea, F.; Goldin, E. The Importance of Intestinal Eotaxin-1 in Inflammatory Bowel Disease: New Insights and Possible Therapeutic Implications. Dig Dis Sci 2016, 61, 1915-1924, https://doi.org/10.1007/s10620-016-4047-z.

31. Khawar, M.B.; Abbasi, M.H.; Sheikh, N. IL-32: a novel pluripotent inflammatory interleukin, towards gastric inflammation gastric cancer, and chronic rhino sinusitis. Mediators of Inflammation 2016, 1, 1-8, https://doi.org/10.1155/2016/8413768.

32. Singh, U.P.; Singh, N.P.; Murphy, E.A.; Price, R.1.; Fayad R.; Nagarkatti, M.; Nagarkatti, P.S. Chemokine and cytokine levels in inflammatory bowel disease patients. Cytokine 2016, 77, 44-49. https://doi.org/10.1016/j.cyto.2015.10.008.

33. Park, J.H.; Peyrin-Biroulet, L.; Eisenhut, M.; Shin, J.I. IBD immunopathogenesis: a comprehensive review of inflammatory molecules. Autoimmunity Reviews 2017, 16, 416-426, https://doi.org/10.1016/j.autrev.2017.02.013.

34. Zhang, L.; Turner, B.; Ribbeck, K.; Ten Hagen, K.G. loss of the mucosal barrier alters the progenitor cell niche via Janus kinase/signal transducer and activator of transcription (JAK/STAT) signaling. J Biol Chem 2017, 292, 21231-21242, https://doi.org/10.1074/jbc.M117.809848.

35. Zeinali, S.; Ghazanfari, S.; Ebrahimi, M.A. Mucin2 gene expression in the chicken intestinal goblet cells are affected by dietary essential oils. Bulg J Agric Sci 2017, 23, 134-141, https://www.researchgate.net/publication/315487151.

36. Lippestad, M.; Hodges, R.R.; Utheim, T.P.; Serhan, C.N.; Dartt, D.A. Resolvin D1 increases mucin secretion in cultured rat conjunctival goblet cells via multiple signaling pathways. Investig Ophthalmol Vis Sci 2017, 58, 4530-4544, https://doi.org/10.1167/iovs.17-21914.

37. Allaire, J.M.; Morampudi, V.; Crowley, S.M.; Stahl, M.; Yu, H.; Bhullar, K.; Knodler, L.A.; Bressler, B.; Jacobson, K. Vallance BA. Frontline defenders: goblet cell mediators dictate host-microbe interactions in the intestinal tract during health and disease. Am J Physiol Gastrointest Liver Physiol 2018, 314, G360-G377, https://doi.org/10.1152/ajpgi.00181.2017.

38. Alsaffar, H.; Martino, N.; Garrett, J.P.; Adam, A.P. Interleukin-6 promotes a sustained loss of endothelial barrier function via Janus kinase-mediated STAT3 phosphorylation and de novo protein synthesis. J Physiol Cell Physiol 2018, 314, C589-C602, https://doi.org/10.1152/ajpcell.00235.2017.

39. Heinrich, P.C.; Behrmann, I.; Haan, S.; Hermanns, H.M.; Müller-Newen, G.; Schaper, F. Principles of interleukin (IL)-6-type cytokine signalling and its regulation. Biochem 2013, 374, 1-20, https://doi.org/10.1042/BJ20030407.

40. Boulanger, M.J.; Bankovich, A.J.; Kortemme, T.; Baker, D.; Garcia, K.C. Convergent mechanisms for recognition of divergent cytokines by the shared signaling receptor gp130. Mol Cell 2003, 12, 577-589, https://doi.org/10.1016/s1097-2765(03)00365-4.

41. Li, Y.; de Haar, C.; Chen, M.; Deuring, J.; Gerrits, M.M.; Smits, R.; Xia, B.; Kuipers, E.J.; van der Woude, C.J. Disease-related expression of the IL6/STAT3/SOCS3 signalling pathway in ulcerative colitis and ulcerative colitis-related carcinogenesis. Gut 2010, 59, 227-235, https://doi.org/10.1136/gut.2009.184176.

42. Silva, I.; Pinto, R.; Mateus, V. Preclinical Study in Vivo for New Pharmacological Approaches in Inflammatory Bowel Disease: A Systematic Review of Chronic Model of TNBS-Induced Colitis. J Clin Med. 2019, 8, 1574, https://doi.org/10.3390/jcm8101574.

43. Arıkan, T.; Akcan, A.; Dönder, Y.; Yılmaz, Z.; Sözüer, E.; Öz, B.; Baykan, M.; Gök, M.; Poyrazoğlu, B. Effects of erythropoietin on bacterial translocation in a rat model of experimental colitis. Turkish journal of surgery, 2019, 35, 202-209, https://doi.org/10.5578/turkjsurg.4272.

https://biointerfaceresearch.com/ 
44. Chidambaram, S,; Thenrajan, R,; Sekharan, B.L.; Pillappan, R. Formulation and evaluation of Prednisolone retention enema as dispersible tablet and vehicle for the treatment of ulcerative colitis. Letters in Applied NanoBioScience 2019, 8, 545-552, https://doi.org/10.33263/LIANBS82.545552. 